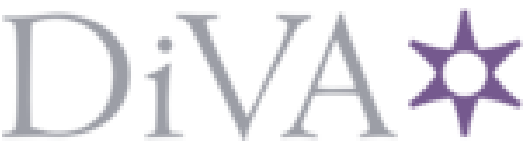

http://www.diva-portal.org

Postprint

This is the accepted version of a paper presented at The International Conference on Frontiers in Handwriting Recognition (ICFHR), September 1-4, 2014, Crete, Greece.

Citation for the original published paper:

Wahlberg, F., Mårtensson, L., Brun, A. (2014)

Scribal Attribution using a Novel 3-D Quill-Curvature Feature Histogram

In: Proceedings International Conference on Frontiers in Handwriting Recognition (ICFHR), 2014

N.B. When citing this work, cite the original published paper.

Permanent link to this version:

http://urn.kb.se/resolve?urn=urn:nbn:se:uu:diva-238270 


\section{Scribal Attribution using a Novel 3-D Quill-Curvature Feature Histogram}

\author{
Fredrik Wahlberg \\ Dept. of Information Technology \\ Uppsala University \\ Email: fredrik.wahlberg@it.uu.se
}

\author{
Lasse Mårtensson \\ Dept. of Business and Economics Studies \\ University of Gävle
}

\author{
Anders Brun \\ Dept. of Information Technology \\ Uppsala University
}

\begin{abstract}
In this paper, we propose a novel pipeline for automated scribal attribution based on the Quill feature: 1) We compensate the Quill feature histogram for pen changes and page warping. 2) We add curvature as a third dimension in the feature histogram, to better separate characteristics like loops and lines. 3) We also investigate the use of several dissimilarity measures between the feature histograms. 4) We propose and evaluate semi-supervised learning for classification, to reduce the need of labeled samples.

Our evaluation is performed on 1104 pages from a $15^{\text {th }}$ century Swedish manuscript. It was chosen because it represents a significant part of Swedish manuscripts of said period. Our results show that only a few percent of the material need labelling for average precisions above $95 \%$. Our novel curvature and registration extensions, together with semisupervised learning, outperformed the current Quill feature.
\end{abstract}

Keywords-writer identification; semi-supervised learning; classification; historical manuscripts;

\section{INTRODUCTION}

The application of computerized writer identification is often associated with signature verification or forensic investigations. However, because vast amounts of historical material is currenlty being digitized this might be one of the more important areas of use in the future. In our libraries, books can be found that no today living person has ever read and few people possess the skill in language and writing styles to do so. Even fewer can separate one scribal hand from another. We set out to attain more knowledge on how the process of large scale scribal attribution can me assisted with computational methods.

We will use the term "scribal attribution" instead of the more common "writer identification" to be more in line with the terminology used in philology. There is also an ambiguity in the word "writer", since it can refer both to the "author" and "scribe," which in the middle ages was only rarely the same person.

Out of the many existing writer identification features, we have chosen to use and extend the so-called Quill feature[1]. It is a feature that is already somewhat tested on historic material, though as a forensic experiment, and suitable for our type of material. Our study is performed on a new data set called C61, containing 1104 pages, and aim to investigate extensions to the current methods and build a parameter free pipeline for scribal attribution.
1) The Manuscript Cod. Ups. C61: The manuscript Cod. Ups. C61 (hereafter C61) is preserved at the University Library in Uppsala. It is a part of the so-called C-collection, which mainly consists of material originating from the monastery in Vadstena, one of the most central scriptorium in medieval Sweden. The manuscript was probably produced in the beginning of the $16^{\text {th }}$ century and it thus belongs to the very end of the medieval period in Sweden. C61 is today composed of several parts that originally did not belong together. In its present state, it consists of 1104 pages written by several scribal hands[2]. A detail of a page is shown in figure 1 .

The script in C61 (all parts) is to classified as cursiva (cursiva recentior; "the younger cursive") according to Albert Derolez' criteria[3]. The distinctive letter forms are a) a single-compartment "a", b) "b, h, k, l" with ascenders with loops and c) " $\mathrm{f}$ " and straight "s" extending below the baseline. Cursiva is to be found in an enormous amount of manuscripts throughout Europe during the $14^{\text {th }}$ and $15^{\text {th }}$ centuries, and in very varying levels of executions. In fact, this style contains a great deal of variation, even though it has proved difficult to divide it into subgroups on geographical or chronological grounds. The most distinct variation is that of the level of execution, as it can vary from very calligraphic version to very informal ones.

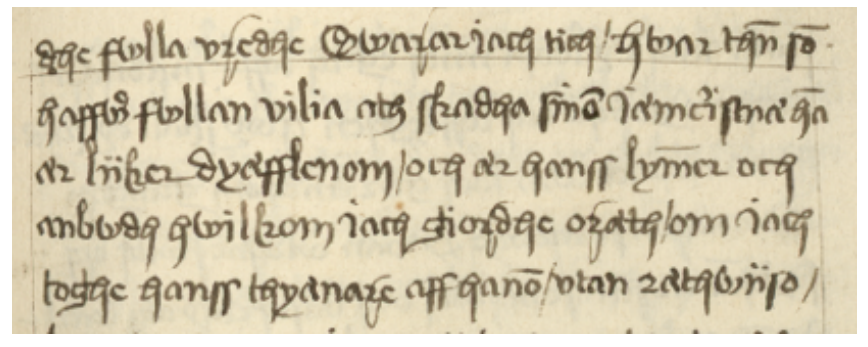

Figure 1. Detail from page 557 of the manuscript C61 used in our study.

2) On Scribal Attribution: Scribal attribution has been performed for a long time in philology. Various criteria have been applied: language forms, orthography, palaeography[4]. Most commonly, palaeography has been looked upon as the most distinctive criteria, whereas language and orthography has been used as complementary evidence. Palaeography 
is thus central in scribal attribution, but also within paleography there has been disagreements as to what features that are distinctive, as to how much variation that one can expect within one hand. To some extent, the material that one is working with sets different conditions for the process of scribal attribution. A formal style, where the letter forms are very homogeneous and the variation between the individual graphs is small, is well suited for an analysis of the morphology, the form of the letter. But if the variation is greater, as is often the case in more quickly executed cursiva, it is difficult to formulate the morphology of the script by one hand close enough for a comparison with another hand to be possible. The most authoritative scribal attribution of C61 is given by [5] and is summarized in table I.

The Quill feature is a very useful way for attacking the cursive, as the focus here mainly is on the variation between thick and thin strokes created by the quill under consideration of the tip angle. This is one way of finding regularity vs variation in the script outside the morphology of the letter forms. An important aspect of the computational methods is that they allow for automated analysis of material with little human input. Thus, it opens for quantitative analyses of medieval script in a way that would be impossible for one individual researcher to perform, though it is of course impossible to dispose of experienced palaeographers in the analysis.

\begin{tabular}{|c|c||c|c||c|c|}
\hline Pages & Hand & Pages & Hand & Pages & Hand \\
\hline $1-129$ & 1 & $425-428$ & 1 & $453-472$ & 1 \\
$130-134$ & 4 & $429-430$ & 2 & $473-476$ & 2 \\
$135-298$ & 1 & $431-438$ & 1 & $477-516$ & 1 \\
$299-304$ & 2 & $439-440$ & 2 & $517-530$ & 2 \\
$305-354$ & 1 & $441-444$ & 1 & $531-534$ & 1 \\
$355-414$ & 2 & $445-446$ & 2 & $535-536$ & 2 \\
$415-422$ & 1 & $447-450$ & 1 & $537-538$ & blank \\
$423-424$ & 2 & $451-452$ & 2 & $539-1104$ & 3 \\
\hline
\end{tabular}

Table I

TABLE SHOWING THE CURRENT STANDARD ATTRIBUTION AS DESCRIBED [5]. THERE IS GENERAL AGREEMENT REGARDING PAGES

1-129 AND 539-1104. PAGES 130-133 IS A MATTER OF DISPUTE.

THERE IS ALSO SOME ARGUMENT ON IF PAGES 137-536 WERE WRITTEN BY TWO HANDS, ONE BEING THE HAND FROM PAGES 1-129.

3) The Quill: The quill pen consisted of a feather, normally from a goose. The feather was hardened and truncated, and finally split at the top. This creates certain conditions for the script where the pen, due to this construction, creates a variation between thicker and thinner strokes. Partly this variation comes from the direction in which the pen is drawn. The thickest strokes are created when the pen is drawn directly downwards in the tip angle that the scribe is holding the pen in, and the thinnest strokes are created when the pen is moved perpendicular to this angle. Another source for variation is that of how heavy pressure that has been put on the pen.

\section{METHOD}

A plethora of different features and approaches exist that quantify the difference between two scribal hands. For reasons outlined above, we have chosen to use and extend the so-called Quill feature proposed in [1]. For a list of other approaches, the writer identification contests at ICFHR 2012 [6] and ICDAR 2013 [7] are good starting points.

The following sections describes a pipeline that was designed to be as parameter-free as possible. Our goal was to evaluate how a large scale scribal attribution system might be constructed to analyse a kind of historical manuscript that is commonly found in Swedish libraries.

\section{A. Pre-processing}

The Quill feature takes a binarized image of text as input. The scanned manuscript images contained spreads of the book C61. The text box identification was performed using projection based techniques, that grew the bounding box from inside an automatically identified text region on each page. The C61 manuscript pages were of good quality with respect to degradation and written on well bleached parchment (a detail from page 557 is shown in figure 1). In contrast to [1], only minor pre-processing was needed. Otsu binarization and minor morphological cleaning (suitable without tuning for any manuscript written with a quill) gave well defined connected components.

For our extensions to the Quill feature, we used a projection based line segmentation technique described in [8]. For each line, a second degree polynomial was fitted to the foreground pixels. This slope estimate was later used to compensate for non-straight lines.

\section{B. Quill feature}

The Quill feature consists of statistics on the angles of the pen tip together with the stroke width at each contour point of every character. The result is a histogram, quantifying the combined effect of the quill pen used and common movement patters of the scribe's hand.

As a first step, the connected components of the binarized image of the manuscript page were found. Contour points of these connected components (8-connected) are then traversed in clock-wise order along the component boundaries. The data collected at each point are value pairs of contour direction and stroke width. In figure 2 , the process of finding measurements for one contour point is shown. Let $\phi_{2}$ be the angle of a vector starting at the contour point currently being processed and ending at the point $r$ steps ahead on the same contour. Analogously, let $\phi_{1}$ be the angle of a vector starting at the contour point $r$ steps behind the current point and ending at the current point. The direction value $\phi$ at the each contour point is defined as an angle perpendicular to the mean of the local $\phi_{1}$ and $\phi_{2}$ pointing into the connected component. 


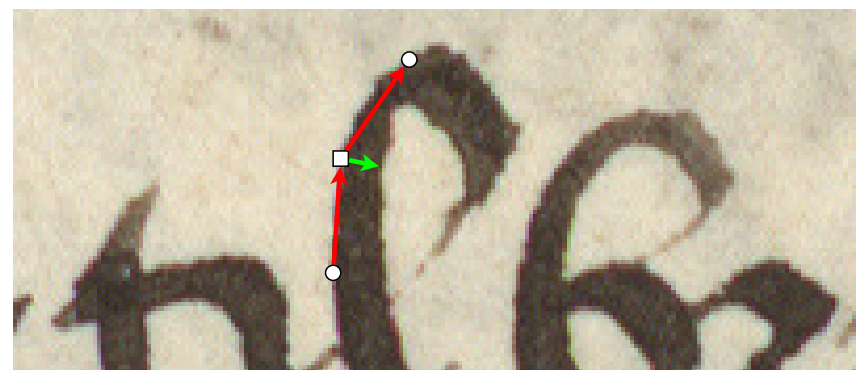

Figure 2. Illustration of the Quill feature processing on one element of an object's contour. The square is the element on the contour that is currently being processed. The red arrows are the "legs" of the processing element with its endpoints at the circles $r$ steps before and after the square on the contour. The green arrow is the estimated direction into the foreground component, perpendicular to the contour direction. The collected value pair is the mean direction of the red arrows together with the length of the green arrow.

The second value of the pair is the stroke width. Here the image is sampled along a Bresenham line at the angle $\phi$ from each contour point. When a background pixel is found along this line, the sampling is stopped and the length of the foreground segment is counted.

A histogram of all value pairs is the final feature, catching stroke widths at different angles. The bins of the histogram was found to work well in [1] with 60 in the angle direction and 40 in the width direction. For a more detailed description of the Quill feature extraction algorithm together with both historical and forensic applications, we refer to [9].

1) Quill feature extensions: A common problem with historical manuscripts is that lines are often tilted and skewed. Even though the text on some pages could be found in a perfect rectangular bounding box, the rectangle would sometimes be tilted up to 10 degrees. Since a normal bin width in the quill histogram might be 6 degrees, this could lead to a shifted histogram for two seemingly identical pages where one is slightly tilted. Also, if the writer would change pen (the quill pens are easily worn out) the stroke width would change. This led to a scaling in the width direction. We propose two compensating extensions of the Quill feature to mitigate these problems. Also, we propose extending the Quill histogram by one dimension to include the curvature of the object contour.

We have tried to compensate for tilted characters using the slope of the line a connected component belonged to. After line segmentation, we fitted second degree polynomials to the pixels of each line to find their respective slopes. This polynomial was used to approximate the mean slope at each point belonging to a connected component. We compensated for the slope at each contour point by subtracting from the $\phi$-value. An alternative would be an affine image transform to straighten the lines. Though that might lead to warped connected components and possible information loss.

To make the Quill feature more invariant to pen width and page rotation, we propose a registration scheme. First,
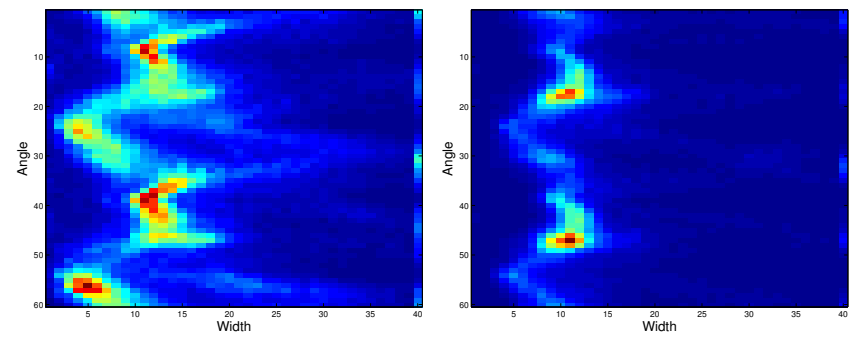

Figure 3. The quill histograms of the pages 20 and 999, belonging to writers 1 and 3 according to [5]. Note how the distribution is more spread in the left histogram. This is because the text is more curvy on the corresponding manuscript page. In the right histogram, the text is less curvy and the two red areas correspond to the many samples of an 11 pixel width in the horizontal direction. The wave structure is visible in both histograms and correspond to the natural differences in stroke width at different angles due to the wide pen tip of the quill pen.

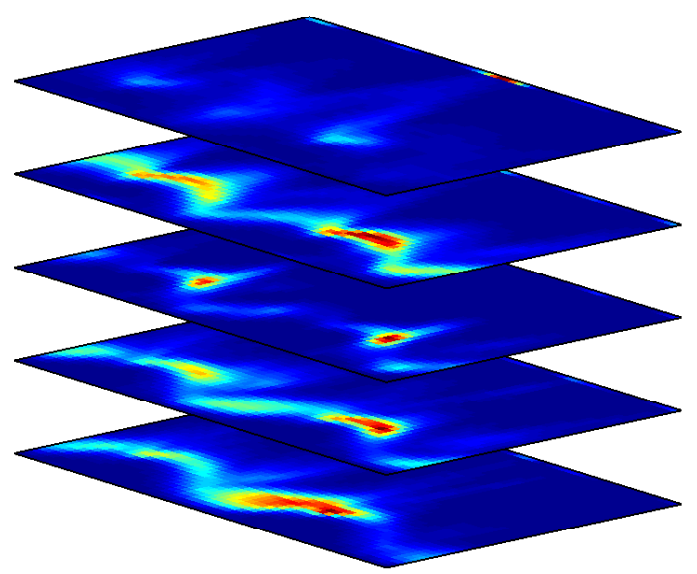

Figure 4. An example of the Quill-Curvature feature histogram as an image stack. Low curvature in the middle image and higher curvature in respective directions to the top and bottom.

the angle component of the value pair is cyclical and it was shifted up to $\pm 10^{\circ}$ (which correspond to moving the histogram image in the vertical direction in figure 3). The second mode in the registration procedure was to allow compensation for different width of the pen tip up to $\pm 25 \%$ (which correspond to stretching the histogram image in the horizontal direction in figure 3). A mean histogram was created from the full stack of all Quill histograms. Each histogram was then registered towards the mean using the $\chi^{2}$ distance (described below) as objective function. A new mean was then created from the stack of registered histogram images and the registration was iteratively improved.

The final extension we propose for the Quill feature is to take curvature along the contour of the connected component into account. We measure an approximation of the contour curvature as the rate of change of the curve normal angle $\phi$, computed by circular convolution with the derivative of a Gaussian. The curvature was added to the quill histogram as a new dimension. This Quill-Curvature histogram was 
given 5 bins for the curvature direction. By taking curvature into account, it was possible to separate similar value pairs sampled different parts of the characters (e.g. at straight lines or stroke loops). An example of a Quill-Curvature histogram as an image stack is shown in figure 4.

\section{Classification}

As stated in [1], most often more complex classifiers are not needed and a kNN classifier is sufficient for writer identification using the Quill feature. Also, in the competitions described in [7] and [6] only a kNN classifier was used. This is very suitable for their purpose since the goal of the competition is to find the best dissimilarity measure. We have implemented this approach as a baseline for evaluating our extensions but also used semi-supervised classification since it fits our purpose better. Our intention is not only writer identification research but also applying the method to a test case for large scale scribal attribution of manuscripts.

Semi-supervised learning (SSL) is a way to reduce the needed training data and have successfully been applied to forensic writer identification[10]. We want to minimize the work of the domain expert and use as few labeled samples as possible, especially for a large scale application. To achieve this, we have implemented an SSL classifier based on graphs[11] where each node represents a Quill histogram (each belonging to a page) and the edge weights are a combination of the dissimilarity metric (with dissimilarity functions as described below) and an additional Gaussian weighting (show in equation 1). The parameter $\sigma$ in equation 1 is determined by maximizing the performance on the training set using leave one out cross validation and $D(\cdot, \cdot)$ is the dissimilarity function.

$$
e(i, j)=1-\exp ^{-\frac{D(\mathbf{v}, \mathbf{u})^{2}}{2 \sigma^{2}}}
$$

With the technique used to produce our manuscript, it is very likely that the performance of a scribe would vary slightly over time (e.g. because of fatigue). In our SSL implementation, the distance between two Quill histograms is defined as the minimum distance path through the graph. That means that a path over 10 incrementally changing pages might be "shorter" than a direct path, with no intermediate steps, to the final destination. The clusters are gradually expanding from the labeled samples creating manifolds corresponding (in the ideal case) to a single scribal hand. For classification, each unlabeled vertex is given the label of the closest labeled vertex on the graph. The cost of the lowest cost path is interpreted as quantifying dissimilarity.

\section{Dissimilarity metrics}

The quill feature gives a histogram describing some characteristics of a manuscript page. It is not obvious how these histograms should be compared. The dissimilarity metric used with success in [1] and [12] is $\chi^{2}$ (chi-square) distance (shown in equation 2). This metric quantifies the difference between two histograms and hence is a distance or dissimilarity metric in our feature space.

$$
D_{\chi^{2}}(\mathbf{v}, \mathbf{u})=\sum_{i=1}^{|v|} \frac{\left(\mathbf{v}_{i}+\mathbf{u}_{i}\right)^{2}}{\mathbf{v}_{i}+\mathbf{u}_{i}}
$$

The $\chi^{2}$ distance in the equation above favors feature elements with small differences. If a more naive approach like Euclidean distance would be used, the larger differences would drown the nuances. It is acknowledged in [1] that other distance metrics might be more suitable but are not explored, citing [13] as a good starting point. We have used two more distance metrics found in [13] that is an excellent survey of metrics to compare probability density functions.

The first new distance function (apart form the $\chi^{2}$ function described above) to be tested was the so-called additive symmetric $\chi^{2}$ distance. It is called additive symmetric because the function's symmetry is achieved by adding two Pearson $\chi$ non-symmetric distance functions.

$$
D_{\operatorname{AddSum\chi } \chi}(\mathbf{v}, \mathbf{u})=\sum_{i=1}^{|v|} \frac{\left(\mathbf{v}_{i}-\mathbf{u}_{i}\right)^{2}\left(\mathbf{v}_{i}+\mathbf{u}_{i}\right)}{\mathbf{v}_{i} \mathbf{u}_{i}}
$$

According to [13], equation 3 is sometimes called "symmetric $\chi^{2}$ divergence," but calls it additive symmetric to set it apart from other symmetric $\chi^{2}$ functions.

The second new distance function is based on entropy and is a symmetric version of the Kullback-Leibler function from [13]. This function is called the Jeffreys divergence and is shown in equation 4. Again the symmetry in 4 is achieved through an addition of two Kullback-Leibler functions. The result is a function measuring relative entropy or information deviation.

$$
D_{\text {Jeffreys }}(\mathbf{v}, \mathbf{u})=\sum_{i=1}^{|v|}\left(\mathbf{v}_{i}-\mathbf{u}_{i}\right) \ln \frac{\mathbf{v}_{i}}{\mathbf{u}_{i}}
$$

Sometimes the inner part of the sums in equations 2 , 3 and 4 can give unusable results. A division by zero or undefined logarithm makes the whole sum undefined. We have for practical reasons simply excluded the undefined parts of the respective sums.

\section{EVALUATION}

\section{A. Evaluation metric}

A commonly used evaluation metric for writer identification is the N-top metric [6]. For each sample in some labeled database, a list of the $\mathrm{N}$ most similar samples are found. If the case of soft $\mathrm{N}$-top, one sample in this list must be of the same writer as the sample currently being investigated to count as a positive hit. The hard N-top demands all entries of the list to have the correct writer. This method evaluates 

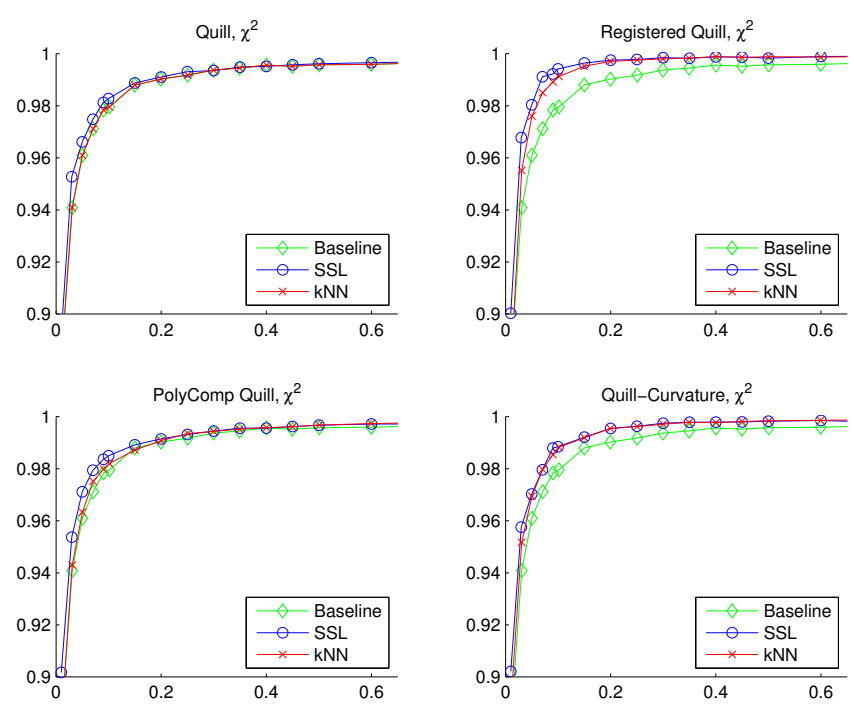

Figure 5. Average precision plotted against the ratio of labeled training for the original Quill feature and three types of extensions. Upper left is the original and upper right is when using registration. Lower left shows results for using polynomial line estimation for compensation and lower right is when using the curvature. The baseline is the original Quill as in [1] and $\chi^{2}$-distance was used as dissimilarity measure .

a feature's property to quantify dissimilarity without taking classification methodology in account.

We have also used average precision as our metric of success. Since the split between the training and evaluation sets were randomized, the evaluation were run multiple times to average out stochastic effects in the precision score. A goal for our study is to determine the needed ratio of training data (assuming similar manuscript material). Hence, we used a more complex classifier than $\mathrm{kNN}$ and evaluated each method with several training data ratios. We also show the needed training data ration to achieve a specified average precision.

\section{B. Results}

Some results for our extensions of the Quill feature are shown in figure 5. The average precision is plotted against the ratio of training data. Each of our extensions lowers the need of training data with Quill-Curvature giving the largest improvements Also, in all but one combination (shown in figure 6) of feature extension and dissimilarity measure the training ration was lowered by the proposed semi-supervised approach.

In table II, some evaluation results are shown. N-top evaluation for each feature/dissimilarity combination does not differ much. For each combination, the best area under the curve (AUC) and required training ration for set result is also presented. The numbers shown are produced from the best found setting of the applicable parameters.

Registered Quill-Curvature with either $\chi^{2}$ or Jeffreys as distance function shows the best performance, both with SSL

\begin{tabular}{|c|c|c|c|c|c|c|}
\hline & \multicolumn{3}{|c|}{ Quill } & \multicolumn{3}{|c|}{ Quill-Curvature } \\
\hline & Orig. & Reg. & Poly. & Orig. & Reg. & Poly. \\
\hline 1-top & 100 & 100 & 100 & 100 & 100 & 100 \\
\hline 5-top & 98 & 99 & 98 & 99 & 99 & 99 \\
\hline 10-top & 94 & 97 & 94 & 97 & 99 & 97 \\
\hline AUC kNN & 98.5 & 99.0 & 98.6 & 98.9 & 99.2 & 98.9 \\
\hline AUC SSL & 98.7 & 99.1 & 98.8 & 98.9 & 99.2 & 99.0 \\
\hline Prec $.95 \mathrm{kNN}$ & 4.0 & 2.8 & 3.6 & 2.9 & 2.4 & 2.9 \\
\hline Prec .95 SSL & 2.9 & 2.3 & 2.8 & 2.7 & 2.2 & 2.5 \\
\hline Prec $.99 \mathrm{kNN}$ & 19.4 & 9.3 & 18.2 & 12.1 & 6.8 & 11.0 \\
\hline Prec .99 SSL & 17.3 & 6.8 & 15.1 & 11.3 & 6.0 & 9.7 \\
\hline
\end{tabular}

RESULTS FOR HARD N-TOP EVALUATION (USING A KNN CLASSIFIER AND $\chi^{2}$-DISSIMILARITY) ON THE FIRST THREE ROWS (NUMBERS IN PERCENT). AUC IS THE AREA UNDER THE CURVE FROM PLOTS OF THE SAME TYPE AS IN FIGURE 5. THE LAST THREE ROWS SHOWS THE TRAINING RATIO NEEDED TO ACHIEVE AN AVERAGE PRECISION OF .99, .95 AND .9 RESPECTIVELY (NUMBERS IN PERCENT). THE LAST FOUR ROWS SHOW RESULTS FROM BOTH USING KNN AND SSL (WITH THE BEST PARAMETERS FOUND).
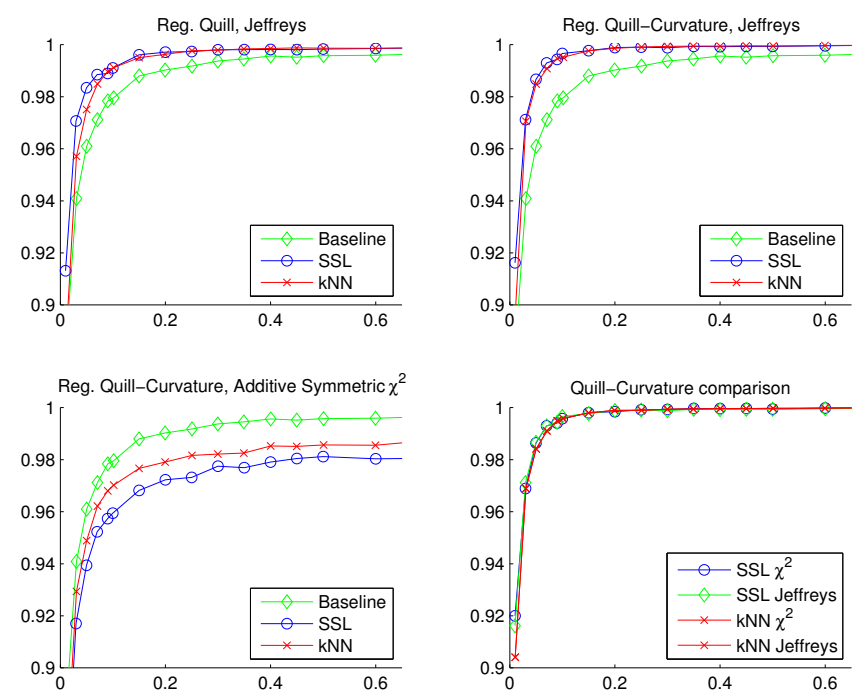

Figure 6. Average precision plotted against training data ratio. The upper plots show results for using the Jeffereys distance function on registered Quill and registered Quill-Curvature. The lower left plot shows results from the less than encouraging additive symmetric $\chi^{2}$-distance. The lower left plot is a comparison between some of the most successful configurations.

and $\mathrm{kNN}$. The additive symmetric $\chi^{2}$ was not suitable as dissimilarity metric as exemplified in figure 6 .

To get a more intuitive view of the feature space, we performed principal component analysis (PCA) on the Quill histograms (shown in figure 7). The cluster for hand 4 in figure 7 is colored blue and consist of four points inside the red and green cluster of hand 1 and 2 . It is quite obvious from the PCA plot (figure 7) that hands 1,2 and 4 are separate form hand 3. Separating the remaining hands proved to be harder. It is not obvious for the feature space if they are one, two or three hands. The maximum distance (original Quill with $\chi^{2}$-distance) between pairs of samples of hand 4 is 0.028 while the minimum distance between any pair with 

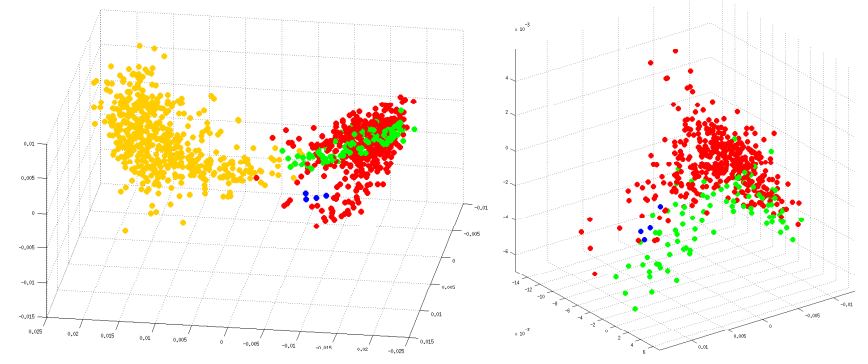

Figure 7. Scatter plot of the most significant three dimensions from a PCA analysis of the Quill histograms. In the left plot, there are two fairly clear clusters. The right plot is the same PCA procedure but only on the right cluster from the left plot. Colours represent authors as defined by [5] and hands have the same colour in both plots.

only one sample belonging to hand 4 is 0.0489 . From this, it could be argued that hand 4 is actually a separate hand from 1 or 2 .

\section{Conclusions}

We propose several performance increasing extensions to the Quill feature. The proposed registration scheme increases accuracy and lowers the need for training data. Also, the proposed addition of curvature to the feature histogram, as a third dimension adding more separation between distinct strokes, increased performance. Of the new distance metrics investigated, Jeffreys was better or on par with $\chi^{2}$. However, additive symmetric $\chi^{2}$ was shown to not be suitable as dissimilarity metric. Finally, the use of semi-supervised learning decreased the need for label training data even more.

In our study, we only used the C61 manuscript. Thus, we can not say how the approach described above would generalize to other types of manuscripts. However, the current class of documents is large (including the historians "gold mine" letter collection "svenskt diplomatarium"). The proposed approach to scribal attribution requires very little work to include more material. The 1104 pages used required little more parameter tuning than specifying that there were two pages per image.

Given the methods used, hand 3 was different than the other hands. Hand 4 is separate and our findings support the claim that this hand is not the same as hands 1 or 2 . However, theses pages were separated by [5] due to letter shapes (a common criterion in paleography). To be able to include this information without human intervention would imply solving the problem of text recognition, which falls far beyond our current scope.

\section{ACKNOWLEDGMENT}

The authors would like to thank Per Cullhed at the Uppsala University library and Zeth Alvered at the department of Scandinavian Languages for their generous help with acquiring the images of C61. This work was funded by
Uppsala University and Vetenskapsrdet grant $\mathrm{nr}$ Dnr 20125743, within the $\mathrm{q} 2 \mathrm{~b}$ - From Quill 2 Bytes initiative, at Uppsala University.

\section{REFERENCES}

[1] A. Brink, J. Smit, M. Bulacu, and L. Schomaker, "Writer identification using directional ink-trace width measurements," Pattern Recognition, vol. 45, no. 1, pp. 162 - 171, 2012.

[2] M. Andersson-Schmitt, H. Hallberg, and M. Hedlund, "Mittelalterliche handschriften der universitätsbibliothek uppsala: Katalog über die c-sammlung. band 1-8," Uppsala, 19881995.

[3] A. Derolez, "The palaeography of gothic manuscript books : from the twelfth to the early sixteenth century," Cambridge, 2003.

[4] L. Mårtensson, "Studier i am 557 4to. kodikologisk, grafonomisk och ortografisk underskning av en isländsk sammelhandskrift från 1400-talet.” Reykjavik, 2011.

[5] G. Klemming, "Heliga birgittas uppenbarelser," Samlingar utgivna av Svenska Fornskriftsällskapet 14:5. Stockholm. Svenska fornskriftsällskapet, 1883-84.

[6] G. Louloudis, B. Gatos, and N. Stamatopoulos, "Icfhr 2012 competition on writer identification challenge 1: Latin/greek documents," in Frontiers in Handwriting Recognition (ICFHR), 2012 International Conference on, Sept 2012, pp. 829-834.

[7] G. Louloudis, B. Gatos, N. Stamatopoulos, and A. Papandreou, "Icdar 2013 competition on writer identification," in Document Analysis and Recognition (ICDAR), 2013 12th International Conference on, Aug 2013, pp. 1397-1401.

[8] F. Wahlberg and A. Brun, "Graph based line segmentation on cluttered handwritten manuscripts," in Pattern Recognition (ICPR), 2012 21st International Conference on, Nov 2012, pp. $1570-1573$.

[9] A. A. Brink, "Robust and applicable handwriting biometrics," Ph.D. dissertation, University of Groningen, 2011.

[10] U. Porwal and V. Govindaraju, "Semi-supervised framework for writer identification using structural learning," Biometrics, IET, vol. 2, no. 4, pp. 208-215, December 2013.

[11] X. Zhu, "Semi-supervised learning with graphs," Ph.D. dissertation, Carnegie Mellon University, 2005.

[12] L. Schomaker and M. Bulacu, "Automatic writer identification using connected-component contours and edge-based features of uppercase western script," Pattern Analysis and Machine Intelligence, IEEE Transactions on, vol. 26, no. 6, pp. 787798, June 2004.

[13] S.-H. Cha, "Comprehensive survey on distance/similarity measures between probability density functions," International Journal of Mathematical Models and Methods in Applied Sciences, vol. 1(4), pp. 300-307, 2007. 